\title{
The LHCb VELO and its use in the trigger *
}

\author{
T. Ruf \\ EP Division, CH-1211 Geneva 23, Switzerland \\ on behalf of the LHCb collaboration
}

\begin{abstract}
Precise vertex reconstruction is a fundamental requirement for the LHCb experiment. Displaced secondary vertices are a distinctive feature of b-hadron decays. The detector setup is presented with special emphasis on its use in the LHCb trigger system together with test results.
\end{abstract}

Key words: LHCb, silicon, vertex detector, vertex trigger

\section{Introduction}

The LHCb experiment [1] is planned to perform systematic studies of CP violation in the beauty sector at the LHC machine by measuring time-dependent decay rate asymmetries using a single arm spectrometer (Fig. 1). This requires the reconstruction of the pp-interaction point and the decay vertex of beauty and charm hadrons with a very good precision. The LHCb VErtex LOcator (VELO) features a series of silicon stations placed along the beam direction. By placing the sensors in a secondary vacuum, one can minimize the material before the first measured point, which is needed to separate the sensor volume from the ultra high vacuum of the LHC machine. The radial distance from the beam is smaller than the aperture required by the LHC during injection and the sensors must therefore be retractable. This is achieved by mounting the stations in a setup similar to Roman pots (Fig. 2).

At the LHC design energy of $\sqrt{s}=14 \mathrm{TeV}$ and the LHCb luminosity of $2 \times 10^{32} \mathrm{~cm}^{-2} \mathrm{~s}^{-1}$, about $200000 \mathrm{~b}$-hadrons are expected to be produced per

‡ Talk presented at the 10th International Workshop on Vertex Detectors, 23-28 September, Brunnen, Switzerland. 


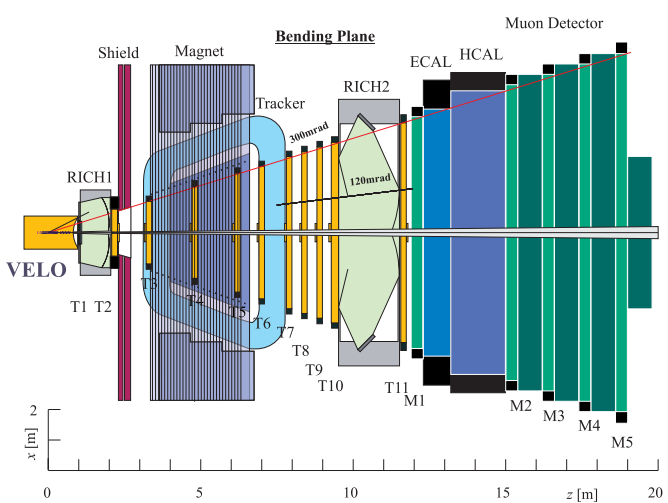

Fig. 1. The LHCb spectrometer seen from above (cut in the bending plane), showing the location of the VELO.

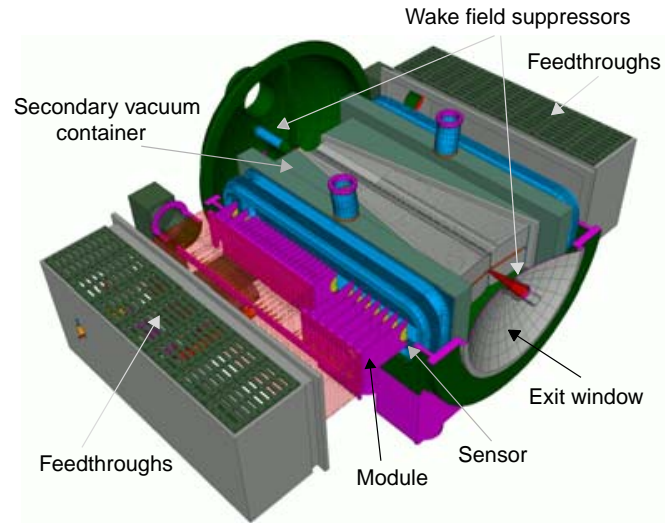

Fig. 2. The VELO Roman pot configuration. One detector half is not installed to show the sensors.

second. However, there are about 30 million proton-proton collisions per second in which no b-hadrons are produced. The task of the LHCb trigger is to select those collisions which contain b-hadrons. Decay vertices displaced from the primary vertex are a good signature for those events. Therefore, the information from the VELO is an important input for the second level trigger. Multiple interactions are rejected by the VETO trigger which uses two dedicated silicon stations upstream of the VELO. The VETO trigger is part of the first level trigger and runs with an input rate of $40 \mathrm{MHz}$.

The first section gives a short overview of the VELO and discusses the sensor design. More details can be found elsewhere [2]. The second section describes the LHCb trigger scheme with special emphasis on the Pile up VETO and Vertex triggers.

\section{VErtex LOcator}

The full coverage of the LHCb acceptance, in the pseudorapidity range of $1.6<\eta<4.9$, is achieved with a series of stations placed along the beam direction. Each station provides an $\mathrm{R}$ and a $\phi$ measurement (Fig. 3). The partial coverage of the backward hemisphere improves the primary vertex measurement. The two first stations upstream are reserved for the pile-up VETO trigger and are only equipped with R-sensors. Some global parameters of the VELO are listed in Table 1. 

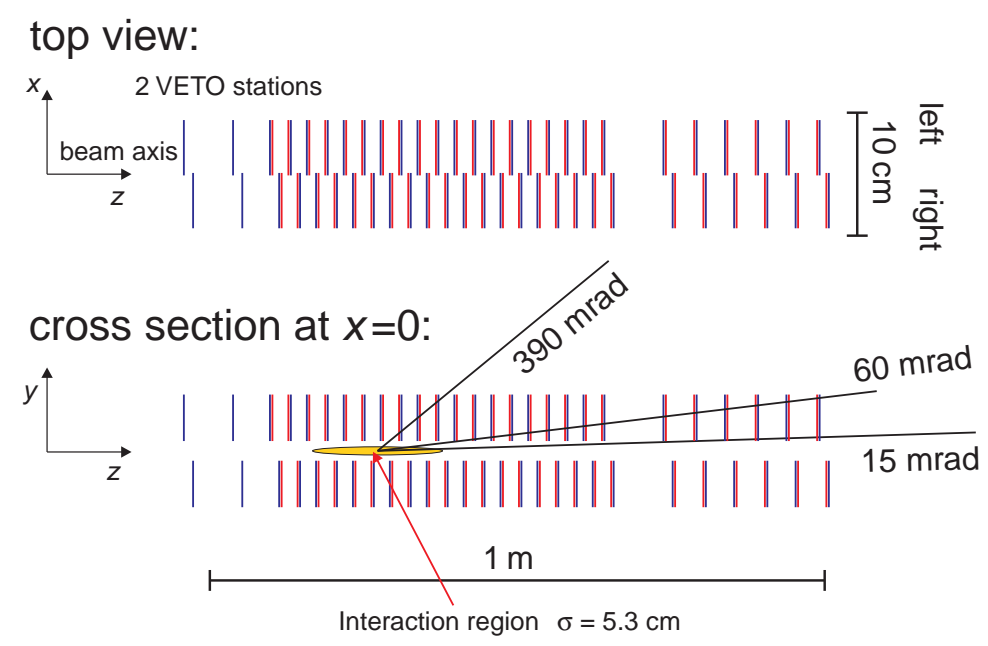

Fig. 3. The top figure shows the VELO setup seen from above. The bottom figure is a cross section of the setup at $x=0$ along the beam axis showing also the nominal position of the interaction area. The three lines indicate the maximum and minimum angular coverage of the VELO and the average angle of tracks in minimum bias events respectively.

\subsection{Sensor design}

The silicon sensors have a semi-circular shape spanning $182^{\circ}$; they are patterned with azimuthal ( $\mathrm{R}$ measuring) or quasi-radial ( $\phi$ measuring) strips (Fig. 4). These views have been chosen in order to optimize the stand-alone tracking performance for the L1 trigger. By using a double metal layer, it is possible to decouple the routing of the signals from the strip geometry and to move most of the electronics out of the acceptance (Fig. 5). The strips in the $\phi$-sensor are tilted with a stereo angle, which is different in sign and magnitude for the inner and outer region. The strips in the R-sensor are segmented into 4 (2) azimuthal sections in the inner (outer) regions. With this design it is possible to determine the primary vertex position in the plane perpendicular to the beam using the R-sensors alone, a feature exploited in the L1 trigger. The pitch varies with radius, making a balance between uniform occupancy and ensuring that the first two points on the track are measured with the finest pitch available.

The LHCb VELO sensors will be subject to a harsh radiation environment. At the innermost radius this will be dominated by charged hadrons and will reach levels of about $10^{14} n_{\mathrm{eq}} / \mathrm{cm}^{2}$ per year (Fig. 6). Due to the $r^{-\alpha}$ dependence of the irradiation, there is a sharp gradient in dose from the inner to the outer radius. The highest radiation levels occur at the place where the sensors have the finest pitch, and the demands on the resolution are greatest. These considerations, combined with extensive prototyping, have led to the choice of n-strip detectors on n-bulk material ( $n$-on- $n$ ), with AC coupling and 
Table 1

Global parameters of the VELO system.

\begin{tabular}{l|c}
\hline number of stations & 25 \\
position of first station upstream & $-17.5 \mathrm{~cm}$ \\
position of last station downstream & $75 \mathrm{~cm}$ \\
total area of silicon & $0.32 \mathrm{~m}^{2}$ \\
total number of channels & 204,800 \\
radiation dose/year at $8 \mathrm{~mm}$ & $(0.5-1.3) \times 10^{14} n_{\mathrm{eq}} / \mathrm{cm}^{2}$ \\
radiation dose/year at $50 \mathrm{~mm}$ & $240 \mathrm{kRad}$ \\
power dissipation & $<1.5 \mathrm{~kW}$ \\
dimensions of the vacuum vessel $($ length $\times \varnothing)$ & $1.8 \mathrm{~m} \times 1 \mathrm{~m}$ \\
\hline
\end{tabular}

polysilicon biasing. It is expected that the sensors have to be replaced every three to four years. The basic parameters of the sensors are listed in Table 2.

Analog readout has been chosen since it provides improved hit resolution, and allows for better monitoring and control of effects due to the very non-uniform radiation damage to the silicon detectors. A total of 128 readout lines will be bonded to a front end chip. Two radiation hard designs are being pursued, one in the $0.25 \mu \mathrm{m}$ CMOS, the other in the DMILL technology [3]. Both chips accept input data at $40 \mathrm{MHz}$ which are kept in an analog pipeline of $4 \mu \mathrm{s}$ latency until the L0 decision is received. Then, 32 channels are eventually read out in $900 \mathrm{~ns}$ in order to cope with the average L0 accept rate of $1 \mathrm{MHz}$. The analog data are sent via twisted pair cables to the L1 electronics situated at $60 \mathrm{~m}$ distance in a radiation free environment. The L1 electronics performs synchronization checks, provides the interface to the L1 trigger and performs zero-suppression and cluster finding. Events accepted by the L1 trigger are processed and transferred to the central DAQ.

\subsection{Silicon RED}

The VELO specific design challenges of,

- varying strip lengths,

- a double metal layer,

- regions of fine pitch and,

- the large and non-uniform irradiation,

have been studied in detail. Several prototype sensors with different technologies have been evaluated, among them double sided sensors, single sided sensors with $p$-on-n-strip readout and oxygenated and non-oxygenated sensors. More details about the $R \& D$ results can be found elsewhere [2]. It turned out that $p$-on- $n$ sensors are much more sensitive to radiation damage than $n$-on$n$ sensors due to the fact that after type inversion the depletion starts from the side opposite to the readout strips. Test-beam results for $p$-on- $n$ sensors 
Table 2

Parameters of the R- and $\phi$-measuring sensors.

\begin{tabular}{lcc}
\hline & R-sensor & $\phi$-sensor \\
\hline readout channels per sensor & 2048 & 2048 \\
smallest (largest) pitch & $40(92) \mu \mathrm{m}$ & $37(98) \mu \mathrm{m}$ \\
length of shortest (longest) strip & $6.4(66.6) \mathrm{mm}$ & $9.2(24.4) \mathrm{mm}$ \\
inner (outer) radius of active area & $8(42) \mathrm{mm}$ & $8(42) \mathrm{mm}$ \\
stereo angle & - & $10^{\circ}-20^{\circ}$ \\
average occupancy in inner (outer) area & $0.5(0.9) \%$ & $0.7(0.5) \%$ \\
\hline
\end{tabular}
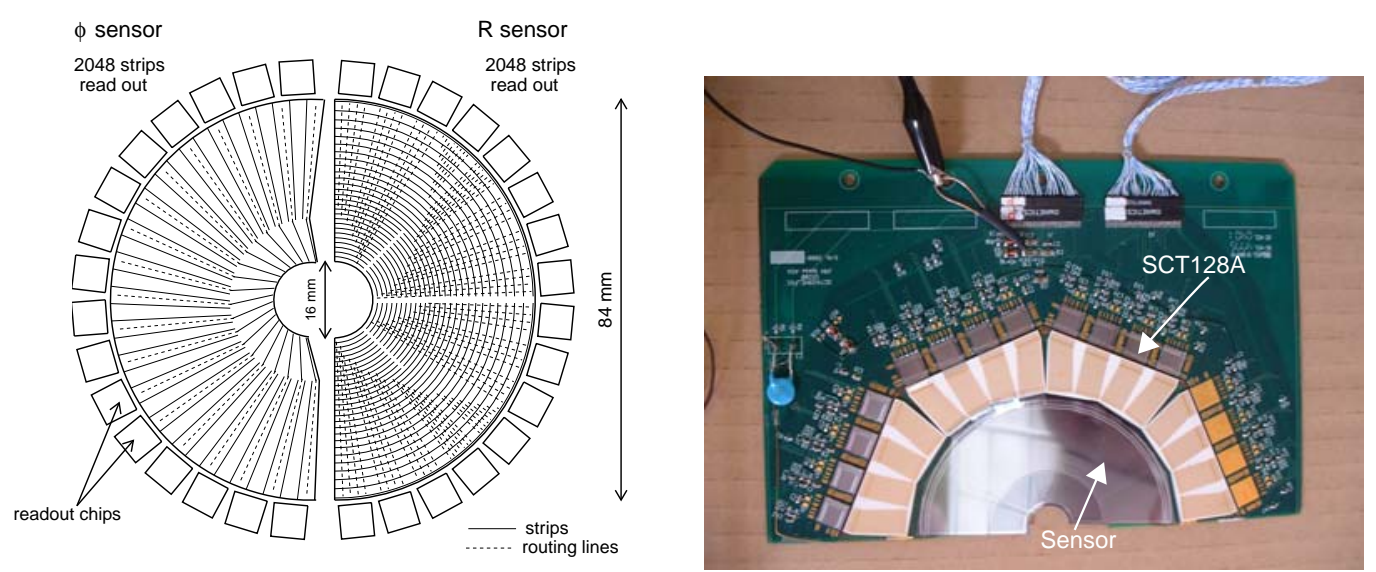

Fig. 4. Schematic view of a R- and Fig. 5. Prototype sensor bonded to $\phi$-measuring sensor. A R-measuring sensor has azimuthal strips at constant radius, whereas a $\phi$-measuring sensor has a prototype hybrid equipped with 12 SCT128A chips.

radial strips with a small stereo angle.

showed a loss of charge to neighbouring strips and the second metal layer, when not fully depleted. This charge loss leads to both, a loss of resolution and efficiency. Whereas results for $n$-on- $n$ sensors showed that operation with up to $40 \%$ under-depletion leads to no loss of efficiency.

\section{LHCb trigger}

The LHCb trigger scheme consists of three basic levels:

L0 The first level trigger is hardware based and operates with a fixed latency of $4 \mu \mathrm{s}$ at an input rate of $40 \mathrm{MHz}$. It looks for high $\mathrm{p}_{\mathrm{t}}$-particles using the electromagnetic and the hadronic calorimeters and the muon system. In addition, there is a pile-up VETO trigger, which rejects events with more than one interaction. The data of the sub-detectors are stored in analog or digital pipelines. After an L0-Yes, all data is digitized and transferred to 
Fig. 6. a) Annual hadron fluences per $\mathrm{cm}^{2}$ normalized to the damage of neutrons of $1 \mathrm{MeV}$ energy, for station 7 and 25 as function of radius. The radial dependence is well described by the function $\mathrm{N} \times r^{-\alpha}$, with $\mathrm{N}$ and $\alpha$ changing as function of $z$. b) The flux per year at $r=0.8 \mathrm{~cm}$ as a function of the station number. c) The parameter $\alpha$ as function of the station number.

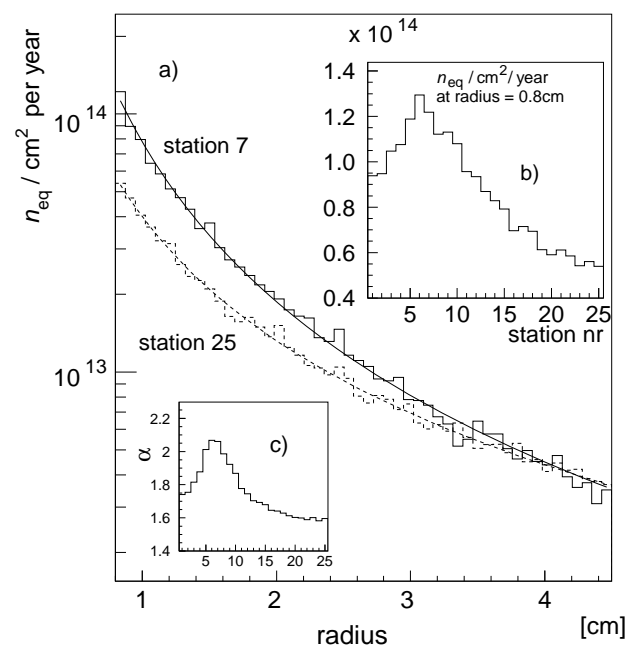

the L1 buffer.

L1 The second level trigger is software based and operates at an input rate of about $1 \mathrm{MHz}$. Up to 1927 events can be stored locally in the sub-detectors L1 buffer. It exploits the VELO information to search for secondary vertices displaced from the primary interaction. After an L1-Yes, data from the individual sub-detectors are sent to the readout network.

L2 \& L3 The higher level triggers have access to all detector information and run on a large CPU farm. Their tasks range from a refined vertex trigger algorithm up to the selection of individual B-decay channels.

\subsection{L1: Vertex trigger}

Selecting events with detached secondary vertices requires the ability to perform tracking and vertex reconstruction using only the VELO information. This is possible, because the occupancy in the VELO is well below $1 \%$ in most areas (Table 2), the efficiency of silicon detectors is high, $>99 \%$, and the VELO is designed to give at least $3 \mathrm{R}$ - and $\Phi$-measurements for a track in the LHCb acceptance. On average, there are only about 1.5 tracks in the innermost $45^{\circ}$-sectors of the R-sensors. The input to the vertex trigger are the $\mathrm{R}$ - and $\phi$-cluster centers. The algorithm consists of five consecutive parts [5]:

- Track finding in the R-projection, efficiency $\eta \approx 98 \%$.

- Calculation of the primary vertex position, $\sigma_{\mathrm{z}}=70 \mu \mathrm{m}, \sigma_{\mathrm{x}, \mathrm{y}}=20 \mu \mathrm{m}$.

- Impact parameter determination for all tracks.

- $3 \mathrm{~d}$ reconstruction of large impact parameter tracks, $\eta \approx 95 \%$.

- Search for two track vertices separated from primary vertex.

The performance of the track finding and vertex reconstruction algorithms were studied with test-beam [4] and agreement with the simulation was found. The execution time of the algorithm (Fig. 7) is well below the maximum 


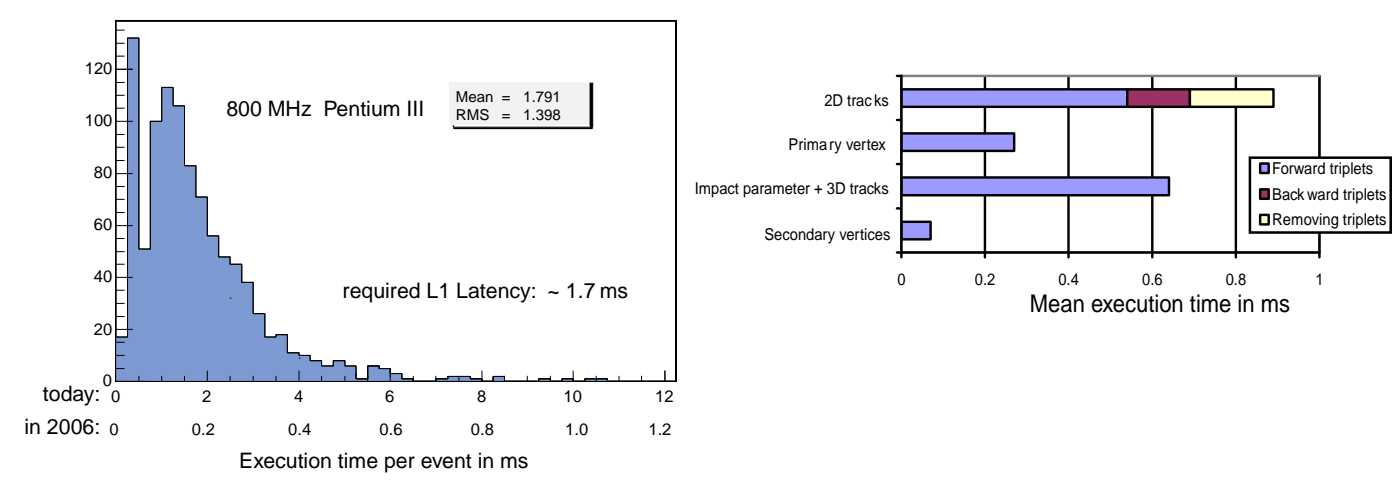

Fig. 7. Left: Execution time of the vertex trigger algorithm. Right: Breakdown in different parts of the algorithm.

latency of about $1.7 \mathrm{~ms}$, assuming that more powerful CPU's become available in 2006 .

Hardware Implementation:

Analog data is digitized, followed by pedestal subtraction and an event by event common mode correction. The addresses of the cluster centers are sent to a CPU-farm via a switch which has to deal with a data rate of $\approx 4$ Gbyte/s and small event fragments of only 170 bytes. The current implementation of the switch and the CPU-farm is centered around a 2d torus topology based on SCI shared memory.

Physics Performance:

Studies for the LHCb Technical Proposal gave efficiencies around 60\% - 80\% depending on the signal channel at a minimum bias event retention of $4 \%$. After tuning the Pythia event generator to reproduce the UA5 and CDF data [6], which increased the charm and b-baryon cross section, the efficiency of the L1algorithm dropped by about a factor of 1.5. The main limitation of the L1 algorithm is the missing momentum information. Tracks with low momentum, and therefore large multiple scattering angles, fake large impact parameter tracks. In addition, the B-decay track signature of having a large $\mathrm{p}_{\mathrm{t}}$ cannot be exploit.

Two proposals exist to overcome this problem:

Super L1 Tries to link tracks with a large impact parameter to the L0 objects: high $\mathrm{p}_{\mathrm{t}}$ Ecal or Hcal clusters or muons. For $\mathrm{B}_{\mathrm{d}}^{0} \rightarrow \pi^{+} \pi^{-}$events, the efficiency to match one of the pions with a VELO track is about $78 \%$, in $\mathrm{B}_{\mathrm{d}}^{0} \rightarrow \mathrm{J} / \psi \mathrm{K}_{\mathrm{s}}^{0}(\mu \mu)$ events, the matching efficiency for one muon is $96 \%$. Using the additional information from the L0 trigger, it has been shown that the loss in performance of the vertex trigger can be overcompensated (Fig. 8). Advantage: Information already exists, amount of data to be send to the L1-farm is very small.

Mini L1 A separate study has shown, that a moderate momentum informa- 
tion of $\sigma\left(\mathrm{p}_{\mathrm{t}}\right) / \mathrm{p}_{\mathrm{t}} \approx 20 \%$ would also be more than sufficient to overcome the L1-TP deficiency (Fig. 8). Disadvantage: Needs a full silicon tracker station close to the magnet. $\sim 30 \%$ more data to be send to the L1-farm.

The final solution for L1 will be described in the Trigger Technical Design Report.

\subsection{Pile Up VETO trigger}

Given the limited amount of time of the L1 trigger, searching for displaced vertices in events which contain more than one pp-interaction is difficult. In addition, the higher occupancy of the Outer Tracker and the two RICH detectors in multiple interaction events degrades the pattern recognition performance. $\mathrm{LHCb}$ decided to tune the luminosity at the $\mathrm{LHCb}$ interaction point for a maximum number of events with single interactions. However, at the $\mathrm{LHCb}$ luminosity of $2 \times 10^{32} \mathrm{~cm}^{-2} \mathrm{~s}^{-1}$, still about $20 \%$ of the events contain multiple interactions with tracks inside the $\mathrm{LHCb}$ acceptance. In order not to occupy the bandwidth of the L0 trigger which is set to $1 \mathrm{MHz}$, the Pile Up VETO trigger [7] rejects those events at level L0.

Two silicon stations only equipped with R-measuring sensors upstream of the VELO stations but inside the same vacuum vessel are dedicated to the Pile Up VETO trigger. Between the two R-measurements, $R_{A}$ and $R_{B}$, of tracks coming from a primary vertex at the position $\mathrm{Z}_{\mathrm{PV}}$ exist the following relation:

$$
\frac{\mathrm{R}_{\mathrm{B}}}{\mathrm{R}_{\mathrm{A}}}=\frac{\mathrm{Z}_{\mathrm{B}}-\mathrm{Z}_{\mathrm{PV}}}{\mathrm{Z}_{\mathrm{A}}-\mathrm{Z}_{\mathrm{PV}}}=\text { const. } \longrightarrow \mathrm{Z}_{\mathrm{PV}}
$$

Without knowing which hits belong to which track, primary vertices still show up as peaks in a histogram of $Z_{\mathrm{PV}}$ for all possible hit combinations.

The Pile Up VETO sensors are readout in binary mode using the BEETLE chip. Histogramming and peak finding is performed in a large FPGA, which needs to have more than $600 \mathrm{I} / \mathrm{O}$ pins and more than $350 \mathrm{k}$ gates. Candidate FPGAs are already identified and more are expected to become available in the coming years.

By rejecting events with multiple interactions at the earliest trigger stage, the conditions of the high $\mathrm{p}_{\mathrm{t}}$ trigger can be relaxed and $(30-40) \%$ more single bb-events are able to pass L0. 


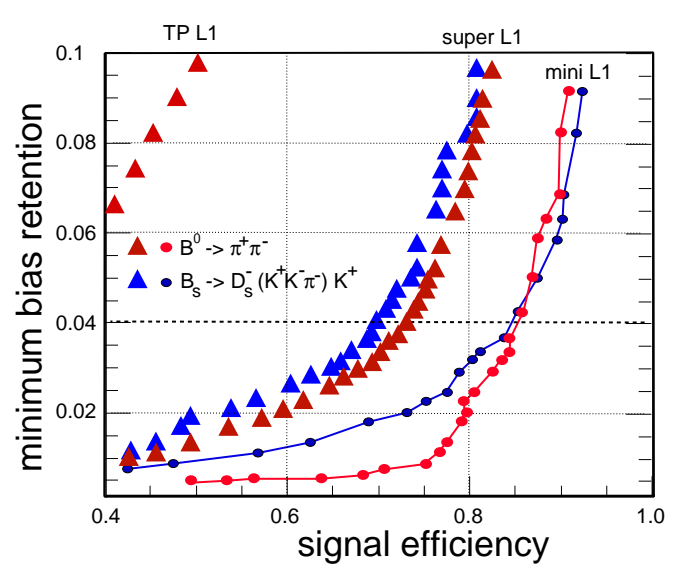

Fig. 8. Minimum bias retention versus signal efficiency for two b-decay channels.

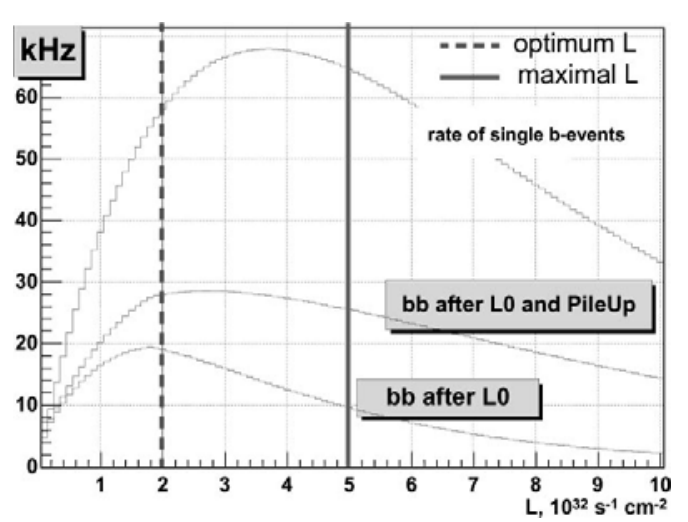

Fig. 9. Performance of the Pile Up Veto trigger.

\section{Conclusion}

In $\mathrm{LHCb}$, silicon detectors are used in the trigger for two different aspects of vertex reconstruction. The Pile Up VETO trigger has the simple task of only counting the number of primary pp-interactions, but it has to work at an input rate of $40 \mathrm{MHz}$. The second level trigger running at an input rate of $1 \mathrm{MHz}$ exploits the full information of the VErtex LOcator in order to perform a standalone track finding and primary and secondary vertex reconstruction. R\&D for the networking and CPU-farm is well advanced. Ideas to overcome the limitations of the algorithm presented in the LHCb Technical Proposal are under study.

\section{References}

[1] LHCb Technical Proposal, CERN/LHCC 98-4.

[2] LHCb VELO Technical Design Report, CERN/LHCC 2001-011.

[3] The Beetle Reference Manual, D. Baumeister et al., LHCb 2001-046;

The SCTA_VELO Reference Manual, J. Buytaert, LHCb 2001-045.

[4] First test-beam results with a prototype of the LHCb vertex detector, F. Teubert, Nuclear Instruments and Methods in Physics Research Section A, Volume 447, Issue 1-2, 2000, pp. 69-75.

[5] The L1 vertex trigger algorithm and its performance, H. Dijkstra and T. Ruf, LHCb note 98-006.

[6] Tuning of Multiple Interactions Generated by Pythia, P. Bartalini et al. LHCb note 99-028. 
[7] The LHCb triggers, N.Y. Zaitsev and L.W. Wiggers, Nuclear Instruments and Methods in Physics Research Section A, Volume 447, Issue 1-2, 2000, pp. 235243 . 